\title{
On the zero viscosity limit in incompressible fluids
}

\author{
A L Mazzucato \\ Department of Mathematics, Penn State University, University Park, PA 16802, \\ U.S.A. \\ E-mail: alm24@psu.edu
}

\begin{abstract}
We discuss the convergence in the limit of vanishing viscosity of solutions of the Navier-Stokes equations for incompressible fluid flow to solutions of the Euler equations in the presence of boundaries. We present explicit examples in 2 and 3 dimensions for which convergence holds in the energy norm, even when the flow is forced through moving boundaries. We obtain convergence rates in viscosity and discuss concentration of vorticity at the boundary in the limit.
\end{abstract}

PACS numbers: 47.27.N-,47.27.nb,47.15.-x,47.27.nd

Keywords: wall-bounded turbulence, incompressible fluid, vanishing viscosity, shear flow, laminar flow. Submitted to: Physica Scripta

\section{Introduction}

In recent years much progress has been made in understanding turbulence in fluid flow. Turbulence in unsteady flows however differs significantly from classical isotropic turbulence. Yet, its mixing properties for example play an important role in many physical processes at different length scales, from stellar dynamics to material behaviour at microscales. From the point of view of mathematical analysis, fundamental open questions remain, especially when boundaries are present. A key question is how to represent mathematically unsteady flow at very high Reynolds number $(R e)$. Onsager [1] suggested that fully developed turbulence is modeled by irregular (weak) solutions of the Euler equations (EE), describing inviscid, incompressible fluids, that dissipate kinetic energy in 3 dimensions or enstrophy in 2 dimensions. This approach may be motivated in part by showing that solutions of the Navier-Stokes equations (NSE), describing viscous, incompressible fluids, converge in an appropriate sense to solutions of the Euler equations as viscosity becomes small. This vanishing or zero viscosity limit has been addressed by several authors, and convergence is known in the whole space (see e.g. the recent paper [2] and references therein). In the case of domains with boundaries, however, there are few rigorous convergence results. The main difficulty 
lies in reconciling the different boundary conditions for viscous and inviscid flows in the limit. In a viscous fluid, velocity is assumed to vanish along any material boundary, that is, no-slip boundary conditions are imposed, while in an inviscid fluid only the normal component of the velocity is assumed to vanish, that is, slip boundary conditions are imposed. At very high $R e$, the flow is well approximated by Euler solutions away from walls, but near boundaries large velocity gradients are expected to develop to fit the no-slip condition. This phenomenon gives rise to a boundary layer, the thickness of which depends on viscosity, where the flow is neither described by the Navier-Stokes nor the Euler equations. The theory of boundary layers, which dates back to Prandtl's pioneering work, is still largely of asymptotic nature (we refer to [3] for an introduction). Unsteady boundary layers represent a key mechanism for mixing, since vorticity can be generated at the walls that may propagate in the bulk, leading to boundary layer separation.

Kato [4] gave a general criterion for the zero viscosity limit to hold, which states that a (weak) solution to NSE on a bounded (smooth) domain converges to a (weak) solution of EE if and only if energy dissipation in a boundary layer of thickness proportional to the viscosity vanishes as viscosity goes to zero (see [5] for an equivalent formulation in terms of enstrophy). This criterion was later generalized by Temam and Wang [6], Wang [7] to assume only control on the dissipation in the normal or tangential direction, but on a slightly larger layer, and allowing Couette-type flows, where the boundary condition is no-slip with respect to a moving boundary. In general it is not known whether weak Navier-Stokes solutions satisfy Kato's criterion. In addition, in 3D we lack a uniqueness theory for weak solutions to NSE and an existence theory for weak solutions to EE. In special situations, convergence in the zero viscosity limit has been shown to hold. In 3D Asano [8], Sammartino and Calfish [9] consider analytic data in a half-space and obtain convergence along with asymptotic estimates for the boundary layer using a modified Cauchy-Kowalewski Theorem (for boundary layer analysis under weaker conditions see also [10]). In 2D, Matsui [11], Bona and Wu [12] show that the vanishing viscosity limit holds for circularly symmetric solutions in a (stationary) disk using respectively the stream function formulations of the equation and explicit Bessel functions expansions. Fluid flow in two dimensions can be viewed as an approximation of flow in thin domains, and it is important for numerical purposes. It is also an exact, though in general unstable, solution to the 3D equations assuming that the velocity is horizontal and independent of the vertical coordinate, i. e., the flow is planar, as for example in the classical case of Couette flow between infinite coaxial, rotating cylinders. Other types of boundary conditions, such as Navier friction, can be considered based on physical principles, which lead to better convergence results $[13,14,15,16,17]$. We also mention the papers [18] and [19], which deal with the zero viscosity limit for linearized NSE respectively in a channel and in the exterior of a disk.

In this paper, we discuss recent work of the author and collaborators [20, 21, 22 ] providing additional examples of flows in two and three dimensions for which convergence in the energy and higher norms as viscosity vanishes and estimates in 
the boundary layer can be rigorously established. We still consider classical unsteady Couette flows, so that NSE reduce to linear equations, but we allow the cylinders to rotate with rough angular velocities. We also consider plane-parallel 3D channel flows, again allowing the channel walls to translate with rough velocities. The symmetry of the problem reduces here NSE to a weakly coupled non-linear system. The rough boundary velocities include impulses and even stochastic motions. In both cases we rewrite the time evolution in the form of an abstract Volterra integral equation and use properties of the fundamental solution of the Stokes equation. We should point out that Kato's criterion easily applies to both circularly-symmetric and plane-parallel flows if the boundary velocities are sufficiently regular, in particular continuous in time. However, even in this case our analysis leads to good estimates on convergence rates in viscosity and a detailed picture of the boundary layer.

One of the key aspects of this work is to isolate the effect of the boundary conditions in highly simplified, but rigorous examples, where symmetry depletes nonlinearities and coupling between different scales. This depletion gives rise to laminar flows for which boundary layer separation does not occur. Nevertheless, even the simplest case of a singular diffusive limit for the heat equation can shed light into the complicated mechanisms governing turbulent fluid flow near boundaries. For example, we rigorously verify the phenomenon of concentration of vorticity at the boundary in the limit. In the circularly symmetric case, we hence show that linear, nearly inviscid flows can produce non-zero torque on the boundary. Furthermore, when the boundary rotates, we also prove rigorously that concentration is affected by tangential acceleration of the boundary. Rapidly varying boundary motions may enhance mixing, and modeling such unsteady Couette flows can then lead to interesting benchmarks for numerical codes at high Re. Furthermore, rotating fluids are relevant in geophysical applications (see $[23,24]$ in the context of zero viscosity limit), and turbulent mixing affects atmospheric and oceanic circulation (e.g. oscillations in the thermohaline circulation).

We conclude with a word on notation. If $\Omega \in \mathbb{R}^{n}$ is an open set, we denote by $L^{p}(\Omega)$, $1 \leq p<\infty$, the space of functions with $p$ th-integrable power, by $L^{\infty}(\Omega)$ the space of (essentially) bounded functions, by $H^{s}(\Omega), s \in \mathbb{R}$, the Sobolev space of functions with $s$ weak derivatives in $L^{2}$. Functions can be scalar or vector valued. By $C$ we mean a generic constant that may change from line to line.

\section{Unsteady Couette flow}

We consider viscous fluid flow inside an infinite vertical cylinder rotating rigidly with angular velocity $\alpha(t)$. We will discuss later the case of flow between coaxial cylinders rotating independently. In this section we denote a point in $\mathbb{R}^{3}$ as $\left(x, x_{3}\right)=\left(x_{1}, x_{2}, x_{3}\right)$ and we identify the cylinder axis with the $x_{3}$ coordinate axis, so that cylindrical coordinates can be written as $\left(r, \theta, x_{3}\right)$ with $r=|x|$. By rescaling we can also reduce to a cylinder with unit radius and we denote its cross section, the unit disk in $\mathbb{R}^{2}$, by $D$. Since the dynamics is for $t>0$, we will always assume that $\alpha(t) \equiv 0$ for $t<0$. 
We assume initially the velocity is planar, circularly symmetric and divergence free, i. e., of the form $v_{0}\left(x_{1}, x_{2}, x_{3}\right)=\left(u_{10}\left(x_{1}, x_{2}\right), u_{20}\left(x_{1}, x_{2}\right), 0\right)$, where the $2 \mathrm{D}$ vector $u_{0}\left(x_{1}, x_{2}\right)=\tilde{u}_{0}(r) x^{\perp}$. By $x^{\perp}$ we mean the vector $\left(-x_{2}, x_{1}\right)$. This assumption also implies that velocity satisfies slip boundary conditions $u_{0} \cdot x=0$ on $\partial D=S^{1}, S^{1}$ denoting the unit circle. For simplicity we take initial data independent of viscosity, although it is not crucial. Then it can be shown that the velocity remains planar, circularly symmetric, and divergence-free with a radial pressure function $p^{\nu}$, for $t>0$. Furthermore, the convection gradient $u^{\nu} \cdot \nabla u^{\nu}$ exactly balances the pressure gradient $\nabla p^{\nu}$, so that the Navier-Stokes equations in the cylinder with no-slip boundary conditions become:

$$
\begin{cases}\partial_{t} u^{\nu}=\nu \Delta u^{\nu}, & \text { for }(t, x) \in(0, \infty) \times D, \\ u^{\nu}(0, x)=u_{0}(x), & \text { for } x \in D, \\ u^{\nu}(t, x)=\frac{\alpha(t)}{2 \pi} x^{\perp}, & \text { for }(t, x) \in(0, \infty) \times \partial D,\end{cases}
$$

where $\Delta=\partial_{x_{1}}^{2}+\partial_{x_{2}}^{2}$ is the Laplace operator in $\mathbb{R}^{2}$, while the Euler equations are simply:

$$
\begin{cases}\partial_{t} u=0, & \text { for }(t, x) \in(0, \infty) \times D, \\ u(0, x)=u_{0}(x), & \text { for } x \in D,\end{cases}
$$

so that any circularly symmetric, divergence-free vector field $u_{0}$ is an exact, steady, 2D Euler solution. Above $\nu$ is the viscosity coefficient proportional to $1 / R e$, if the equations are nondimensionalized. We stress we do not assume $u_{0}(x)=\alpha(0)(2 \pi)^{-1} x^{\perp}$ at the boundary (as in e.g. [12]). This distinction is relevant when $\alpha$ is discontinuous at the origin and gives rise to an initial layer for $u^{\nu}$ at $t=0$ that has an effect on vorticity production and rates of convergence in the zero viscosity limit.

For Couette flow, the zero viscosity limit reduces to the limit $u^{\nu} \rightarrow u_{0}$ as $\nu \rightarrow 0$. The most natural norm in which to take this limit is the energy norm:

$$
\left\|u^{\nu}\right\|_{L^{\infty}\left([0, T], L^{2}(D)\right)}^{2}=\sup _{0<t<T} \int_{D}\left|u^{\nu}(x, t)\right|^{2} d x, \quad 0<T<\infty
$$

since pairing NSE with $u$ and integrating by parts gives that $\left\|u^{\nu}(T)\right\|_{L^{2}(D)}^{2}-\left\|u_{0}\right\|_{L^{2}(D)}^{2}$ equals the total energy dissipation on the interval $[0, T]$.

We assume first $\alpha$ is smooth; in particular, $\alpha(0)=0$. We also assume that the initial data is smooth, since its regularity does not affect the vanishing viscosity limit for the velocity, though it affects the behaviour of the pressure gradient and vorticity. We pass to a rotating frame by introducing a forcing term in the equation and integrate in time to obtain

$$
\begin{aligned}
& u^{\nu}(t)=e^{\nu t \Delta} u_{0}+\mathcal{S}^{\nu}(\alpha)(t), \\
& \mathcal{S}^{\nu}(\alpha)(t)=\int_{[0, t)}\left(I-e^{\nu(t-s) \Delta}\right) \frac{x^{\perp}}{2 \pi} \alpha^{\prime}(s) d s .
\end{aligned}
$$

Here $e^{\nu t \Delta}$ is the solution operator of the heat equation $\partial_{t} u^{\nu}=\nu \Delta u^{\nu}$ in $D$ with homogeneous boundary conditions. It forms a semigroup for $t \geq 0$ which is continuous in $t$ with values in $L^{q}(D), 1 \leq q<\infty$, but only bounded in $L^{\infty}(D)$. It is also continuous 
in the Sobolev spaces $H^{s}(D), s \geq 0$ (see e.g. [25, Chapter 15, Section 1]). Formula (3b) extends then to angular velocities for which the weak derivative $\alpha^{\prime}$ gives rise to a finite (Radon) measure $\alpha^{\prime}(s) d s=d \alpha$, that is, for all $\alpha$ of bounded variation on $\mathbb{R}$. This is the case if the rotation of the boundary is started or stopped impulsively, since then $\alpha^{\prime}$ is a Dirac distribution. We denote the space of functions of bounded variation on an interval $I$ by $\mathrm{BV}(I)$ with norm

$$
\|\alpha\|_{\mathrm{BV}(I)}=\|\alpha\|_{L^{1}(I)}+\int_{I} d \alpha(s)
$$

and recall that BV functions have left and right limits at every point (see e.g. [26, Section 3.5]). Formula (3b) allows us to treat also stochastic rotations (for details we refer to [21]). Since $e^{\nu t \Delta} f=0$ on $\partial D$ for any $t>0$ and any square-integrable function $f, \mathcal{S}^{\nu}(\alpha)(t)$ represents the contribution from the rotation of the boundary and enforces the boundary condition even for irregular angular velocities. In fact, for $|x|=1$ :

$u^{\nu}(x, t)=\mathcal{S}^{\nu}(\alpha)(t)=\int_{[0, t)} \frac{x^{\perp}}{2 \pi} d \alpha(s)=\left[\lim _{s \rightarrow t_{-}} \alpha(s)-\lim _{s \rightarrow 0_{-}} \alpha(s)\right] \frac{x^{\perp}}{2 \pi}=\alpha\left(t_{-}\right) \frac{x^{\perp}}{2 \pi}$.

From $(3 a-3 b)$ and continuity of the heat semigroup, it follows easily that $u^{\nu} \rightarrow u_{0}$ as $\nu \rightarrow 0_{+}$in the energy norm. Informally, the limit holds in the energy norm in spite of the different boundary conditions imposed on $u^{\nu}$ and $u_{0}$, since restriction to the boundary is not a meaningful operation for $L^{2}$ functions, which are defined only up to sets of zero area. Using properties of $e^{\nu t \Delta}$ in more regular Sobolev norms and rescaling, we estimate the rate of convergence of $u^{\nu}$ to $u_{0}$ as $\nu \rightarrow 0_{+}$:

$$
\begin{aligned}
\left\|u^{\nu}(t)-u_{0}\right\|_{L^{2}(D)} & \leq\left\|\left(e^{\nu t \Delta}-1\right) u_{0}\right\|_{L^{2}(D)}+\|\alpha\|_{\mathrm{BV}([0, t])} \sup _{s \in[0, t]}\left\|\left(e^{\nu s \Delta}-1\right) \frac{x^{\perp}}{2 \pi}\right\|_{L^{2}(D)} \\
& \leq C(t) \nu^{\tau / 2}\left(\left\|u_{0}\right\|_{H^{\tau}(D)}+\|\alpha\|_{\mathrm{BV}([0, t])}\left\|\frac{x^{\perp}}{2 \pi}\right\|_{H^{\tau}(D)}\right),
\end{aligned}
$$

for all $0<\tau<1 / 2$. To reconcile the boundary conditions in the limit, we use that functions in the Sobolev space $H^{s}$ do not have a restriction to the boundary, as long as $s<1 / 2$ (see e.g [27, Chapter 1, Section 11]).

We go even further and allow for angular velocity in $L^{p}([0, \infty)), 1 \leq p<\infty$, by integrating $(3 b)$ by parts and approximating the angular velocities with smooth $\alpha$ 's :

$$
\begin{aligned}
& u^{\nu}(t)=e^{\nu t \Delta} u_{0}+\mathcal{S}^{\nu}(\alpha)(t), \\
& \mathcal{S}^{\nu}(\alpha)(t)=-\lim _{\varepsilon \searrow 0} \nu \int_{0}^{t-\varepsilon} \Delta e^{\nu(t-s) \Delta} \frac{x^{\perp}}{2 \pi} \alpha(s) d s,
\end{aligned}
$$

where the limit exists at least in $L^{2}(D)$, uniformly in $t \in[0, T]$. In both $(3 b)$ and $(5 b)$, we need to ensure that no-slip boundary conditions continue to be satisfies for $\nu>0$. This is clear for $\alpha$ smooth and follows from the continuity of $u^{\nu}$ in the radial direction by approximation, if $\alpha$ is not smooth: $u^{\nu}(x, t) \rightarrow \alpha(t) \frac{x^{\perp}}{2 \pi}, \quad$ as $\quad|x| \rightarrow 1$, weakly in time $t$ and angle $\theta$ (in the space $H^{-1}([0, \infty) \times \partial D)$ ).

To control $\mathcal{S}^{\nu}(\alpha)(t)$ we need further estimates in Sobolev norms. We employ the smoothing property of the heat semigroup $e^{\nu t \Delta}$ for $t>0$, that is, it maps $H^{\tau}$ to $H^{\sigma}$, 
$\tau>\sigma$, with bound (see again [25, Chapter 15]): $\left\|e^{\nu t \Delta} f\right\|_{H^{\tau}} \lesssim(\nu t)^{-(\tau-\sigma) / 2}\|f\|_{H^{\sigma}}$. This bounds yields the following estimates for $\mathcal{S}^{\nu}(\alpha)(t)$ :

$$
\begin{aligned}
& \left\|\mathcal{S}^{\nu}(\alpha)(t)\right\|_{H^{\sigma}(D)} \leq \int_{0}^{t}\left\|\nu \Delta e^{\nu(t-s) \Delta} \frac{x^{\perp}}{2 \pi} \alpha(s)\right\|_{H^{\sigma}(D)} d s \leq\|\alpha\|_{L^{p}([0, t])} \\
& \cdot\left(\int_{0}^{t}\left\|\nu \Delta e^{\nu s \Delta} \frac{x^{\perp}}{2 \pi}\right\|_{H^{\sigma}(D)}^{p^{\prime}} d s\right)^{1 / p^{\prime}} \leq C(t) \nu^{(\tau-\sigma) / 2}\|\alpha\|_{L^{p}([0, t])}\left\|\frac{x^{\perp}}{2 \pi}\right\|_{H^{\tau}(D)}
\end{aligned}
$$

provided that $0 \leq \sigma<\tau<1 / 2$ and $p>4$, where $p^{\prime}=(p-1) / p$ denotes the conjugate exponent to $p$. We also used that $x^{\perp} / 2 \pi$ is smooth on $D$. Selecting $\sigma=0$ above establish convergence $u^{\nu} \rightarrow u_{0}$ as $\nu \rightarrow 0_{+}$in the energy norm for $\alpha \in L^{p}([0, T]), p>4$, with again a rate of convergence:

$$
\left\|u^{\nu}(t)-u_{0}\right\|_{L^{2}(D)} \leq C(t) \nu^{\tau / 2}\left(\left\|u_{0}\right\|_{H^{\tau}(D)}+\|\alpha\|_{L^{p}([0, t])}\left\|\frac{x^{\perp}}{2 \pi}\right\|_{H^{\tau}(D)},\right),
$$

with $0<\tau<1 / 2$. The restriction $p>4$ can be removed by considering other classes of Sobolev spaces and weaker norms [21]. Finally, using that for circularly symmetric velocities, $r \nabla p^{\nu}=\left|u^{\nu}\right|^{2} x / r$, the above established convergence translates to convergence for the pressure

$$
r \nabla p^{\nu}(t) \rightarrow r \nabla p_{0} \quad \text { in } \quad H^{\sigma}(D), \quad \forall 0<\sigma<1 / 2 .
$$

We now turn to analyzing the flow in the boundary layer. We first establish that there is no boundary layer separation by proving that $u^{\nu}$ converges to $u_{0}$ uniformly in space and time, in particular pointwise, in the interior of $\Omega$ away from the boundary, which follows from known properties of the heat equation on a bounded domain. Next, to get a more precise picture in the boundary layer, we observe that $V^{\nu}(t, x)=\left(1-e^{\nu t \Delta}\right) x^{\perp} / 2 \pi, t>0$ (=0 otherwise), solves:

$$
\begin{aligned}
& \partial_{t} V^{\nu}=\nu \Delta V^{\nu} \quad \text { on } \quad \mathbb{R} \times D \\
& V^{\nu}(x, t)=\chi_{\mathbb{R}^{+}}(t) \frac{x^{\perp}}{2 \pi}=g(t, x), \quad \text { on } \quad[0, \infty) \times \partial D,
\end{aligned}
$$

which is a boundary value problem for the heat equation and can be analyzed via boundary integrals in terms of the Green's function. Given $h$ supported in $\mathbb{R}^{+} \times \partial D$, we set

$\mathcal{D}^{\nu} h(t, x)=\int_{0}^{\infty} \int_{\partial D} h(s, y) \frac{\partial H}{\partial n_{y}}(\nu(t-s), x, y) d S(y) d s, \quad t \in \mathbb{R}, x \in D$,

where $H(\nu t, x, y)=(4 \pi \nu t)^{-1} e^{-|x-y|^{2} / 4 \nu t} \chi_{\mathbb{R}^{+}}(t)$, and $n_{y}$ is the unit outward normal to $\partial D$ at $y$. Then,

$$
\left|V^{\nu}(t, x)-2 \mathcal{D}^{\nu} g(t, x)\right| \leq C \nu^{1 / 2} t^{1 / 2}, \quad \forall x \in \bar{D}, \quad t>0 .
$$

Since $\mathcal{S}^{\nu} \alpha(t)=\int_{0}^{t} V^{\nu}((t-s), x) d \alpha(s)$, it then follows that

$$
\mathcal{S}^{\nu} \alpha(t)=2 \int_{0}^{t} \mathcal{D}^{\nu} g((t-s), x) d \alpha(s)+\mathcal{R}_{\nu}(t)
$$

with $g$ defined in (9) and

$$
\left\|\mathcal{R}_{\nu}(t)\right\|_{L^{\infty}(D)} \leq C(t) \nu^{1 / 2}\|\alpha\|_{\mathrm{BV}([0, t])} .
$$


In particular, (11) and (12) imply that the boundary layer is of thickness $O\left(\nu^{1 / 2}\right)$ as expected in a laminar flow.

We conclude this section with a discussion of vorticity concentration at the boundary in the limit. This phenomenon is perhaps the most interesting from the point of view of unsteady turbulence and turbulent mixing. Although symmetry prevents boundary layer separation, our analysis nevertheless rigorously shows that the boundary alone generates vorticity and vorticity production is affected by how irregular in time the motion of the boundary is (see also the recent preprint [28]). We recall that vorticity is the curl of the velocity, and in $2 \mathrm{D}$ curl $=\operatorname{div}^{\perp}$, so that the vorticity can be identified with a scalar. We first observe that vorticity production can only happen in a layer near the boundary of vanishing thickness as $\nu \rightarrow 0$. At the same time, we cannot have $\omega^{\nu}=\operatorname{curl} u^{\nu} \rightarrow \omega_{0}=\operatorname{curl} u_{0}$, since informally the integral of vorticity over $D$ depends on the value of the velocity at the boundary and the initial velocity is generally not assumed to satisfy the boundary conditions. Mathematically, we therefore expect $\omega^{\nu} \rightarrow \omega_{0}+\mu$ as $\nu \rightarrow 0_{+}$where $\mu$ is a measure supported on the boundary. $\mu$ can be thought of as a vortex sheet, although technically there is no flow outside of $D$.

To make this argument rigorous, we exploit circular symmetry to derive boundary conditions for $\omega^{\nu}$. We assume temporarily that $\alpha$ is smooth and deal with rougher angular velocities by approximation. Then, Stokes' Theorem immediately gives

$$
\int_{D} \omega^{\nu}(t, x) d x=\alpha(t), \quad \forall t>0,
$$

from which it also follows that $\omega^{\nu}$ solves

$$
\begin{cases}\partial_{t} \omega^{\nu}=\nu \Delta \omega^{\nu}, & \text { for } x \in(0, \infty) \times D, \\ x \cdot \nabla \omega^{\nu}(t, x)=\frac{\alpha^{\prime}(t)}{2 \pi \nu}, & \text { for } x \in(0, \infty) \times S^{1},\end{cases}
$$

Next, we show the total vorticity regardless of its sign is uniformly bounded as $\nu \rightarrow 0$, which implies (by the Riesz-Markov Theorem, see e.g. [29]) that $\omega^{\nu}$ must converge to a measure on the closed unit disk $\bar{D}$. This bound can be achieved by approximating the absolute value $\left|\omega^{\nu}\right|$ with $\phi_{\epsilon}\left(\omega^{\nu}\right), \phi_{\varepsilon}$ convex smooth functions. By passing to the limit $\varepsilon \rightarrow 0$ and using (14) we have

$$
\left\|\omega^{\nu}(t)\right\|_{L^{1}(D)} \leq\left(\|\alpha\|_{\mathrm{BV}([0, t])}+\left\|\omega_{0}\right\|_{L^{1}(D)}\right) .
$$

Finally, combining (13), (14), and (15) with strong convergence $\omega^{\nu} \rightarrow \omega_{0}$ in the interior gives, as a weak-* limit in the space of finite (Radon) measures on $\bar{D}$ :

$$
\omega^{\nu}(t) \rightarrow \omega_{0}-\left(\int_{D} \omega_{0}(x) d x-\alpha(t-)\right) \frac{d \theta}{2 \pi}, \quad \nu \searrow 0_{+}
$$

That is, we rigorously prove that there is concentration of vorticity at the boundary in the vanishing viscosity limit if either there is non-zero total vorticity initially or the boundary accelerates tangentially for some $t>0$ (recall we assumed $\alpha(t)=0$ for all $t<0$ ), or both. (16) thus provides explicit examples of linear, nearly inviscid flows that produce a non-zero torque on the boundary. 
We mention that the results of this section extend to unsteady Couette flow between cylinders rotating independently with rough angular velocities $\alpha_{j}, j=1,2$ [21]. In this case, the Navier-Stokes equations reduce to a heat equation on an annulus $\mathcal{A}=\left\{x \in \mathbb{R}^{2}: \rho<|x|<1\right\}, 0<\rho<1$, and the solution can be written as $u^{\nu}=e^{\nu t \Delta} u_{0}+\mathcal{S}^{\nu}\left(\alpha_{1}, \alpha_{2}\right)(t)$ with

$\mathcal{S}^{\nu}\left(\alpha_{1}, \alpha_{2}\right)(t)=\int_{[0, t)}\left(I-e^{\nu(t-s) \Delta}\right)\left[\frac{\frac{x^{\perp}}{2 \pi}-\rho^{2} \frac{x^{\perp}}{2 \pi|x|^{2}}}{1-\rho^{2}} d \alpha_{1}(s)-\frac{\rho^{2}\left(\frac{x^{\perp}}{2 \pi}-\frac{x^{\perp}}{2 \pi|x|^{2}}\right)}{1-\rho^{2}} d \alpha_{2}(s)\right]$.

For vorticity concentration, we have similarly to (16):

$\omega^{\nu}(t) \rightarrow \omega_{0}-\left(\int_{\{|x|=1\}} \tilde{u}_{0}(1) d \theta-\alpha_{1}(t-)\right) \frac{d \theta}{2 \pi}+\left(\int_{\{|x|=\rho\}} \tilde{u}_{0}(\rho) d \theta-\rho \alpha_{2}(t-)\right) \frac{d \theta}{2 \pi}$,

weakly-* in the space of (Radon) measures on $\bar{D}$, where $\tilde{u}_{0}(r) x^{\perp}=u_{0}(x), \rho \leq r \leq 1$.

\section{Plane-parallel channel flow}

The analysis of the zero viscosity limit for Couette flow can be carried out also for 3D plane-parallel flow in a channel where the walls translate independently with irregular velocities. In this case, there is still coupling, though weak, between different components of the velocity, so that this example can be a point of departure for a more challenging study of fully non-linear flows with symmetry, such as flows generated by a rotating sphere. By rescaling, we can reduce to consider a channel of unit width, which we write in the form $\Omega=\{(x, y, z): 0 \leq z \leq 1\}$. The velocity has symmetry $u^{\nu}(t, x, y, z)=\left(v^{\nu}(t, z), w^{\nu}(t, x, z), 0\right)$, which is preserved under NS evolution and makes $u^{\nu}$ automatically divergence free. To simplify the analysis, we also assume periodic boundary conditions in horizontal directions, i.e., we work on $\mathcal{O}=\{(x, z): x \in \mathbb{R} / \mathbb{Z}, z \in[0,1]\}$. In this case, the solution to NSE and EE is unique. We first assume that the walls move in the $x$ direction with the same rough velocity $\alpha \in L^{p}([0, \infty)), 1 \leq p<\infty$, and discuss the case of arbitrary velocities in the $(x, y)$ plane later. Then NSE reduce to the weakly coupled, non-linear system:

$$
\begin{cases}\partial_{t} v^{\nu}=\nu \partial_{z}^{2} v^{\nu}, & \text { for } t>0, z \in[0,1], \\ \partial_{t} w^{\nu}+v^{\nu} \partial_{x} w^{\nu}=\nu \Delta w^{\nu}, & \text { for } t>0,(x, z) \in \mathcal{O}, \\ v^{\nu}(z, t)=\alpha(t), \quad w^{\nu}(x, z, t)=0, & \text { for } t>0, z=0,1,\end{cases}
$$

where $\Delta=\partial_{x}^{2}+\partial_{z}^{2}$. We again assume that the initial data is regular and independent of viscosity $u_{o}(x, y, t)=(V(z), W(x, z), 0)$. The solution of $\mathrm{EE}$ is then $u(x, z, t)=$ $(v(z, t), w(x, z, t), 0)$ with

$$
v(t, z)=V(z), \quad w(t, x, z)=W(x-t V(z), z) .
$$

A solution formula similar to $(3 b)$ holds for $v^{\nu}$, where $(2 \pi)^{-1} x^{\perp}$ is simply replaced by the constant function 1 and $\Delta$ by $\partial_{z}^{2}$, and $w^{\nu}$ solves a Volterra integral equation:

$$
w^{\nu}(t)=e^{t\left(\nu \Delta-V \partial_{x}\right)} W+\int_{[0, t)} e^{(t-s)\left(\nu \Delta-V \partial_{x}\right)}\left[\left(V-v^{\nu}(s)\right) \partial_{x} w^{\nu}(s)\right] d s .
$$


Estimates similar to (6) continue to hold for the convergence of $v^{\nu}$ to $v$, which in turn lead to the convergence $w^{\nu} \rightarrow w$, and consequently $u^{\nu} \rightarrow u$, as $\nu \rightarrow 0_{+}$in the energy norm. Here we use that $\partial_{x} w^{\nu}$ solves the same equation as $w^{\nu}$ plus the maximum principle. In fact, similarly to (7) we obtain the rate of convergence [22]:

$$
\sup _{0<t<T}\left\|u^{\nu}(t)-u(t)\right\|_{L^{2}(\mathcal{O})} \leq C(T) \nu^{\tau / 2}\left(\left\|u_{0}\right\|_{H^{\tau}(\mathcal{O})}+\|1\|_{H^{\tau}([0,1])}\|\alpha\|_{L^{p}([0, T]}\right),
$$

where $0<\tau<1 / 2, p>4$.

To analyze the boundary layer, we use here odd reflections across the walls, i. e., the method of images, and the explicit formula for solutions to the heat equation (in $z)$. For simplicity of exposition we assume $V \equiv 1$ and refer to [22] for the general case. Then, we obtain:

$$
\left|V(z)-v^{\nu}(z, t)\right| \leq C(t)\left(\|\alpha\|_{L^{1}(\mathbb{R})}+1\right) \psi\left((t \nu)^{-1 / 2} \delta(z)\right)
$$

where $\delta(z)=\min (1-z, z)$ and $\psi$ is rapidly decreasing . At the same time, (20) and the positivity of the fundamental solution for the heat equation give

$$
\begin{aligned}
& \left(w^{\nu}-w\right)(t, x, z)=\left[e^{t \nu \Delta}-1\right] W(x-V(z) t, z)+R^{\nu}(t, x, z), \\
& \left.\left|R^{\nu}(t, x, z)\right| \leq C(t)\left\|\partial_{x} W\right\|_{L^{\infty}(\mathcal{O})} \int_{[0, t)} e^{(t-s) \nu \Delta} \mid V(z)-v^{\nu}(s, z)\right) \mid d s
\end{aligned}
$$

Consequently, from (21) and (22) it follows that $u^{\nu} \rightarrow u$ uniformly in $0<t<T$ and $x$

as $\nu \rightarrow 0$, as long as $\delta(z)>\eta(\nu)$ with $\frac{\nu^{1 / 2}}{\eta(\nu)}=o(1)$ as $\nu \rightarrow 0_{+}$. In particular, there is no boundary layer separation and the boundary layer has thickness $O\left(\nu^{1 / 2}\right)$, as expected since the flow is laminar.

We conclude by mentioning that the above analysis can be extended to more general translations of the walls in the $x, y$ plane $\left(\alpha_{j}(t), \beta_{j}(t), 0\right), j=1,2$, by replacing $\alpha(t) 1$ in the integral equations for $v^{\nu}$ and $w^{\nu}$ with linear functions of the form:

$$
\Psi(t, z)=\left[\gamma_{2}(t)-\gamma_{1}(t)\right] z+\gamma_{2}(t)
$$

\section{Acknowledgments}

The author wishes to thank her adviser and collaborator Michael Taylor, collaborators Milton Lopes Filho and Helena Nussenzveig Lopes, colleague Victor Nistor for the insight they provided into this work. The author also thanks Jin Kelliher for useful discussions. This work was partially supported by the US National Science Foundation through grants DMS-0405803 and DMS-0708902.

\section{References}

[1] L Onsager. Statistical hydrodynamics. Nuovo Cimento (9), 6(Supplemento, 2(Convegno Internazionale di Meccanica Statistica)):279-287, 1949.

[2] N Masmoudi. Remarks about the inviscid limit of the Navier-Stokes system. Comm. Math. Phys., 270(3):777-788, 2007. 
[3] H Schlichting and K Gersten. Boundary-layer theory. Springer-Verlag, Berlin, enlarged edition, 2000. With contributions by Egon Krause and Herbert Oertel, Jr., Translated from the ninth German edition by Katherine Mayes.

[4] T Kato. Remarks on zero viscosity limit for nonstationary Navier-Stokes flows with boundary. In Seminar on nonlinear partial differential equations (Berkeley, Calif., 1983), volume 2 of Math. Sci. Res. Inst. Publ., pages 85-98. Springer, New York, 1984.

[5] J P Kelliher. On Kato's conditions for vanishing viscosity. Indiana Univ. Math. J., 56(4):17111721, 2007.

[6] R Temam and X Wang. On the behavior of the solutions of the Navier-Stokes equations at vanishing viscosity. Ann. Scuola Norm. Sup. Pisa Cl. Sci. (4), 25(3-4):807-828 (1998), 1997. Dedicated to Ennio De Giorgi.

[7] X Wang. A Kato type theorem on zero viscosity limit of Navier-Stokes flows. Indiana Univ. Math. J., 50(Special Issue):223-241, 2001. Dedicated to Professors Ciprian Foias and Roger Temam (Bloomington, IN, 2000).

[8] K Asano. Zero-viscosity limit of the incompressible Navier-Stokes equation. II. Sürikaisekikenkyūsho Kōkyūroku, (656):105-128, 1988. Mathematical analysis of fluid and plasma dynamics, I (Kyoto, 1986), Part I unpublished.

[9] M Sammartino and R E Caflisch. Zero viscosity limit for analytic solutions, of the Navier-Stokes equation on a half-space. I. Existence for Euler and Prandtl equations. Comm. Math. Phys., 192(2):433-461, 1998.

[10] M C Lombardo, M Cannone, and M Sammartino. Well-posedness of the boundary layer equations. SIAM J. Math. Anal., 35(4):987-1004 (electronic), 2003.

[11] S Matsui. Example of zero viscosity limit for two-dimensional nonstationary Navier-Stokes flows with boundary. Japan J. Indust. Appl. Math., 11(1):155-170, 1994.

[12] J L Bona and J Wu. The zero-viscosity limit of the 2D Navier-Stokes equations. Stud. Appl. Math., 109(4):265-278, 2002.

[13] T Clopeau, A Mikelić, and R Robert. On the vanishing viscosity limit for the 2D incompressible Navier-Stokes equations with the friction type boundary conditions. Nonlinearity, 11(6):1625$1636,1998$.

[14] M C Lopes Filho, H J Nussenzveig Lopes, and G Planas. On the inviscid limit for two-dimensional incompressible flow with Navier friction condition. SIAM J. Math. Anal., 36(4):1130-1141 (electronic), 2005.

[15] P B Mucha. The Eulerian limit and the slip boundary conditions - admissible irregularity of the boundary. In Regularity and other aspects of the Navier-Stokes equations, volume 70 of Banach Center Publ., pages 169-183. Polish Acad. Sci., Warsaw, 2005.

[16] W M Rusin. On the inviscid limit for the solutions of two-dimensional incompressible NavierStokes equations with slip-type boundary conditions. Nonlinearity, 19(6):1349-1363, 2006.

[17] Y Xiao and Z Xin. On the vanishing viscosity limit for the 3D Navier-Stokes equations with a slip boundary condition. Comm. Pure Appl. Math., 60(7):1027-1055, 2007.

[18] M C Lombardo and M Sammartino. Zero viscosity limit of the Oseen equations in a channel. SIAM J. Math. Anal., 33(2):390-410 (electronic), 2001.

[19] M C Lombardo, R E Caflisch, and M Sammartino. Asymptotic analysis of the linearized NavierStokes equation on an exterior circular domain: explicit solution and the zero viscosity limit. Comm. Partial Differential Equations, 26(1-2):335-354, 2001.

[20] L C Lopes Filho, A L Mazzucato, and H J Nessenzveig Lopes. Vanishing viscosity limit for incompressible flow inside a rotating circle. To appear in Physica D.

[21] L C Lopes Filho, A L Mazzucato, H J Nessenzveig Lopes, and M E Taylor. Vanishing viscosity limits and boundary layers for circularly symmetric 2d flows. To appear in Bull. Braz. Math. Soc. (N.S.).

[22] A L Mazzucato and M E Taylor. Vanishing viscosity plane parallel channel flow and related singular perturbation problems. To appear in Analysis and PDE. 
[23] E Grenier and N Masmoudi. Ekman layers of rotating fluids, the case of well prepared initial data. Comm. Partial Differential Equations, 22(5-6):953-975, 1997.

[24] N Masmoudi. The Euler limit of the Navier-Stokes equations, and rotating fluids with boundary. Arch. Rational Mech. Anal., 142(4):375-394, 1998.

[25] M E Taylor. Partial differential equations. III, volume 117 of Applied Mathematical Sciences. Springer-Verlag, New York, 1997. Nonlinear equations, Corrected reprint of the 1996 original.

[26] G B Folland. Real analysis. Pure and Applied Mathematics (New York). John Wiley \& Sons Inc., New York, second edition, 1999. Modern techniques and their applications, A Wiley-Interscience Publication.

[27] J-L Lions and E Magenes. Non-homogeneous boundary value problems and applications. Vol. I. Springer-Verlag, New York, 1972. Translated from the French by P. Kenneth, Die Grundlehren der mathematischen Wissenschaften, Band 181.

[28] J P Kelliher. Vanishing viscosity and the accumulation of vorticity on the boundary. Preprint arXiv:0805.2402.

[29] M Reed and B Simon. Methods of modern mathematical physics. I. Academic Press Inc. [Harcourt Brace Jovanovich Publishers], New York, second edition, 1980. Functional analysis. 